\title{
Convergence of Quantum Dot-Barcodes with Microfluidics and Signal Processing for Multiplexed High-Throughput Infectious Disease Diagnostics
}

Jesse M. Klostranec, Qing Xiang, Gabriella Farcas, Jeongjin A. Lee, Alex Rhee, Erin I. Lafferty, Steve Perrault, Kevin C. Kain, Warren C. W. Chan*

\author{
SUPPORTING INFORMATION \\ Section S1 (Methods \& Procedure): Pages 1 to 12 \\ Section S2 (Figures): Pages 13 to 14 \\ Section S3 (Movie): Page 15 \\ Section S4 (Tables): Pages 16 to 19 \\ Section S5 (Cost Analysis): Page 20
}

\section{SECTION S1 (METHODS \& PROCEDURES)}

\section{Quantum Dot Synthesis}

CdSe core $\mathrm{ZnS}$ capped quantum dots (Qdots) were synthesized using organometallic methods described previously (S1-S4). Briefly, $12-20 \mathrm{~g}$ of tri- $n$-octylphosphine oxide (TOPO, 98\% pure, Sigma Aldrich, St. Louis, MO) was heated in a three neck flask to $150^{\circ} \mathrm{C}$ under $\mathrm{Ar}$ gas. $160 \mu \mathrm{L}$ of dimethylcadmium (97\%, Strem Chemicals, Newburyport, MA) was injected and mixed in with the heated TOPO for $\sim 15$ minutes. After three purges under vacuum, the contents of the three neck flask were heated to $350^{\circ} \mathrm{C}$. A 2 molar precursor solution of selenium (Se powder, 99.5\%, Sigma Aldrich) and tri- $n$-octylphosphine (TOP, Sigma Aldrich) was then injected into the three neck and the temperature quickly lowered to $300^{\circ} \mathrm{C} . \mathrm{Cd}: \mathrm{Se}$ ratios in the ranges of 1.5:1 to 2.5:1 were used. Qdot emission was tracked during the growth phase by measuring the emission profile of aliquots of the solution in the three neck flask using a fluorimeter (FluoroMax-3, Jobin Yvon Horiba, Edison, NJ). Once the desired peak emission wavelength had been reached, capping precursor solution consisting of diethyl zinc (Sigma 
Aldrich), hexamethyldisilathiane $\left(\mathrm{TMS}_{2}(\mathrm{~S})\right.$, Sigma Aldrich) and TOP was injected into the three neck drop wise at a rate of $\sim 1 \mathrm{~mL} / \mathrm{min}$.

Following Qdot capping, the three neck temperature was lowered to $<60^{\circ} \mathrm{C}$ and chloroform was added. Several washes with methanol and chloroform (in a 2:1 ratio) were used to precipitate out nanoparticles from unreacted precursors. The final TOPO coated Qdots were stored in chloroform until use.

\section{Quantum Dot Barcode Synthesis}

Qdot barcodes (QdotBs) were prepared using methods described previously (S5-S6). Briefly, $5 \mu \mathrm{m}$ diameter polystyrene microbeads (Bangs Laboratories, Fishers, IN) with carboxylic acid functional groups on the surface were swollen in propanol and TOPO-coated Qdots in chloroform were added (roughly $1.5 \times 10^{7}$ beads in $1 \mathrm{~mL}$ of propanol and $<100 \mu \mathrm{L}$ of Qdots in chloroform). Owing to hydrophobic-hydrophobic interaction, the Qdots diffused into the microbead interior. The incubation lasted 1 hour for QdotB1 (570 nm emitting Qdots only) and QdotB2 (615 nm emitting Qdots only) samples, while for QdotB3, the incubation was split into two steps with $570 \mathrm{~nm}$ emitting Qdots added for the whole hour incubation and $615 \mathrm{~nm}$ emitting Qdots added only for the second half hour. The samples were washed several times (between 7-10) with propanol and stored in a fridge at $4^{\circ} \mathrm{C}$ until used for an assay. The interval of time between bead preparation and the start of an assay did not exceed $12 \mathrm{hrs}$.

\section{Microchannel Fabrication}

Microchannel fabrication followed standard soft lithography techniques (S7-S9). Photomasks of the desired microchannel pattern were prepared using AutoCAD software (San 
Rafael, CA) and printed on a transparency by the Photoplot Store (Colorado Springs, CO). The resolution of the print was $1.59 \mu \mathrm{m}$ (the distance between two pixels).

Fabrication of the masters began by spin coating a $15 \mu \mathrm{m}$ thick layer of 2015 series SU8 photoresist (MicroChem Corp., Newton, MA) on 3.5 inch diameter Si wafers (Virginia Semiconductor, Fredericksburg, VI) and prebaking the samples. Each wafer then had the photomask laid on top of the photoresist, ink surface down, and was exposed to $365 \mathrm{~nm}$ UV light at a power density of $35 \mathrm{~mW} / \mathrm{cm}^{2}$ for a duration of $\sim 4$ seconds using a SUSS MA6 mask aligner (SUSS MicroTec Inc., Waterbury Center, VT). Following standard postbaking procedures, the wafers were immersed in SU8 Nanodeveloper (MicroChem Corp.) for $\sim 1$ minute to dissolve away any photoresist not exposed to the UV light. The masters were then washed with isopropanol and dried with compressed $\mathrm{N}_{2}$ gas.

The polydimethylsiloxane (PDMS) prepolymer kits (RTV 615, General Electric Silicones, Wilton, CT) used come in two parts; part A is the prepolymer; part B contains a crosslinker. Prepolymer was mixed in a 10A:1B ratio. Masters were placed in pyrex Petri dishes and $22 \mathrm{~g}$ of prepolymer was poured on top of each. The samples were then placed under vacuum for $\sim 2 \mathrm{hrs}$ to degas (remove bubbles from) the prepolymer. A 3 hour incubation followed in an oven set at $80^{\circ} \mathrm{C}$.

Once removed from the oven, the cured PDMS slabs were peeled off the masters and excess polymer around the outside of the microchannel pattern was removed. A single master had patterns for two polymer microchannels. The surfaces of the PDMS substrates and glass coverslips (170 $\mu \mathrm{m}$ thick, VWR, Mississauga, ON) were then carefully cleaned using scotch tape. Both PDMS substrates and glass coverslips were loaded into the chamber of a plasma cleaner (Harrick Plasma, Ithaca, NY) and exposed to oxygen plasma for 1 min. Immediately 
after, the surfaces of the PDMS and coverslips were brought into contact to irreversibly seal the two substrates together. Double distilled water was dispensed into the microchannels to keep the channel surfaces hydrophilic. Finally, small pieces of glass were placed on top of the channel wells to keep the water from evaporating, enabling long term storage of the samples.

\section{Quantum Dot Barcode Assay}

\subsection{Antigen Coating of QdotBs}

QdotBs prepared in propanol were vortexed, sonicated for 10 seconds and then run through a $5 \mathrm{~mL}$ filter (Falcon, VWR). Samples initially suspended in $1 \mathrm{~mL}$ of propanol at a concentration of $1.5 \times 10^{7}$ beads $/ \mathrm{mL}$ were split into $250 \mu \mathrm{L}$ aliquots and centrifuged at $8000 \mathrm{rpm}$ for 3 minutes. The supernatant was aspirated and the QdotBs were resuspended in $100 \mu \mathrm{L}$ of 0.1 M MES buffer (pH 5.5). Two more washes of the beads with MES buffer were completed and the samples were then resuspended in $90 \mu \mathrm{L}$ of MES buffer. A stock solution of $0.0092 \mathrm{~g} \mathrm{~N}$ dimethylaminopropyl- $N$ '-ethylcarbodiimide (EDC, Sigma Aldrich) in $1 \mathrm{~mL}$ MES buffer was prepared and $5 \mu \mathrm{L}$ were added to each sample. Samples were then incubated on a vortex, inducing a light shake, for 15 minutes.

Following the EDC incubation, samples were centrifuged at $9000 \mathrm{rpm}$ for 3 minutes and aspirated. A wash with $100 \mu \mathrm{L}$ of MES buffer followed, with centrifugation again at $9000 \mathrm{rpm}$. An antigen solution was prepared at a concentration of $34.4 \mu \mathrm{g} / \mathrm{mL}$ in carbonate-bicarbonate buffer ( $\mathrm{pH}$ 9.4). The antigens used were hepatitis B surface antigen (HBsAg, Advanced Immunochemical, Long Beach, $\mathrm{CA})$, non-structural protein $4\left(\mathrm{NSP}_{4}\right.$, Advanced Immunochemical) and glycoprotein 41 (gp41, Advanced Immunochemical) for hepatitis B virus (HBV), hepatitis C virus (HCV) and human immunodeficiency virus (HIV), respectively. The 
diluted antigen stock solutions were added to the samples to a final volume of $100 \mu \mathrm{L}$ followed by a 15 minute incubation on a vortex.

After incubation with antigen solution, samples were centrifuged at $6500 \mathrm{rpm}$ for 3 minutes, then aspirated. The QdotBs were then resuspended in $100 \mu \mathrm{L}$ of quenching buffer (50 mM Glycine and $0.1 \%$ Tween) and incubated for another 15 minutes on a vortex. Following this incubation, samples were centrifuged at $5500 \mathrm{rpm}$ for 3 minutes, aspirated and resuspended in $100 \mu \mathrm{L}$ of $3 \%$ milk in phosphate buffer saline (PBS). A subsequent incubation on a vortex for 30 minutes served to block the QdotBs with milk proteins. Finally, the QdotBs were washed one more time with TRIS wash buffer ( $\mathrm{pH} \mathrm{8.0),} \mathrm{using} \mathrm{centrifugation} \mathrm{at} 5000 \mathrm{rpm}$. This sample could be stored dry over night if necessary (however, this was never done for these experiments).

\subsection{Target Antibody Capture Using QdotBs}

Stock solutions of target antibody solutions were prepared in human serum. For HBV, clone X12 anti-HBsAg was used (Advanced Immunochemical), for HCV clone 8A1 anti-HCV NS-4 was used (Biodesign International, Saco, ME) and for HIV, clone 5A1 anti-HIV-1 gp41 was used (Biodesign International). Antigen-coated QdotBs were resuspended in spiked human serum samples to a final volume of $100 \mu \mathrm{L}$. They were then incubated on a vortex for 15 minutes, followed by two washes using TRIS wash buffer, centrifuging samples at $5000 \mathrm{rpm}$.

A stock solution of AlexaFluor-488 dye conjugated goat anti-mouse IgG antibodies (Invitrogen, Burlington, ON) was diluted 1:300 in TRIS wash buffer. $100 \mu \mathrm{L}$ of this solution was used to resuspend each sample. Samples were covered in tinfoil (to prevent organic dye photobleaching) and placed on a vortex for 15 minutes. Two final washes of the QdotBcomplexes using $100 \mu \mathrm{L}$ of TRIS wash buffer were completed before resuspending the samples in $500 \mu \mathrm{L}$ of TRIS wash buffer for short term storage. 


\subsection{Multiplexed Assays}

For the multiplexed assays, antigen-coated QdotBs were prepared as described in section 4.1. All experiments used approximately the same number of total beads during antibody capture. If there were two types of antigen QdotBs being used, then half the microbeads in a sample corresponded to one code, while the rest corresponded to the other. The same method was used for samples that used three different QdotBs.

For the incubation of QdotBs with target antibody-spiked human serum, a total volume of $100 \mu \mathrm{L}$ was always used. Therefore, if a sample was incubated with two different targeting antibodies, then $50 \mu \mathrm{L}$ of each spiked serum solution were added. Similarly, if three different targeting antibodies were to be incubated, $33 \mu \mathrm{L}$ of each solution were added.

Figure 4B lists, which antigen-coated QdotBs were incubated with which target antibodyspiked human serum samples. For control (no target antibody) samples, human serum with no target antibodies was added instead. The rest of the target antibody capture assay followed the methods described in section 4.2 .

\section{Commercial ELISA kit assay}

FDA-approved ELISA kits were purchased from Biorad (Hercules, CA) and the instructions given with the kits were followed exactly. All solutions used, except for the samples of human serum spiked with antibodies, were provided with the kits.

Briefly, ELISA plates with antigens conjugated to the surfaces were taken out of a fridge to warm to room temperature. For the HIV kit (Genetic Systems HIV-1/HIV-2 PLUS O EIA), $25 \mu \mathrm{L}$ of diluent was added to each well along with $75 \mu \mathrm{L}$ of sample or control. For the HBV kit (Monolisa Anti-HBs 3.0), samples and controls were added directly. Three reps were completed 
for each concentration value and control sample. A plastic plate sealer was applied and the plates were incubated in a dry incubator at $37^{\circ} \mathrm{C}$ for $1 \mathrm{hr}$.

After the incubation, the plate was washed 5 times using the supplied wash buffer (diluted 1:30 times from the stock solution), with 30 second intervals taken between washes. $100 \mu \mathrm{L}$ of conjugate solution (containing antigens conjugated to horseradish peroxidase, HRP) was then added to each well and a plate sealer applied. A 30 minute incubation followed in the $37^{\circ} \mathrm{C}$ oven followed by another 5 washes with wash buffer. $100 \mu \mathrm{L}$ of a solution containing $o$ phenylenediamine (OPD) wash then added to each well. HRP catalyzes the conversion of OPD to 2,3-diaminophenazine (DAP), which changes the absorption characteristics of the solution (S10-S11). Following this, the reaction was stopped after 30 minutes using a supplied solution. The change in optical density was measured using a plate reader, with results given as the difference in absorption measurements between a peak at $450 \mathrm{~nm}$ and negligible absorption in the range of $615-630 \mathrm{~nm}$. Results for the ELISA kits are shown in Figures 3B and 3C and Tables $S 3$ and $S 4$.

\section{Detection Experiments}

First, QdotB complexes in $500 \mu \mathrm{L}$ of TRIS wash buffer were centrifuged at $4000 \mathrm{rpm}$ for 3 minutes and aspirated. They were then resuspended in $30 \mu \mathrm{L}$ of double distilled water.

Microchannels were flushed with double distilled water once before use, by filling the buffer and waste wells and applying suction at the sample well using a custom tool. Fluid was removed from all wells prior to the introduction of sample into the chip.

$20 \mu \mathrm{L}$ of sample were loaded into the sample well, followed by $20 \mu \mathrm{L}$ of double distilled water into each of the buffer and waste wells. The microfluidic chip was then aligned on the stage of an inverted epiflourescent microscope (IX71, Olympus, Center Valley, PA) and 
immersion oil was applied to the lens of a $60 \mathrm{X}$ objective (1.35 NA, Olympus). The objective lens was brought into focus at the entrance of the sample well. Electrodes were placed in each of the wells, leads connected to the outputs of a voltage regulation circuit (see Figure $S 2$ ). The input of the voltage regulation circuit was connected to a high voltage power supply (CZE1000R, Spellman High Voltage Electronics Corp., Hauppauge, NY), which supplied 300V and $60 \mu \mathrm{A}$ to the regulation circuit during a typical experiment. The voltage ratio between the buffer and sample channels was set at 1.8 .

Once QdotB complexes started to flow into the microchannel, the objective lens focus was moved to align with the flow focusing sample stream located downstream from the intersection of the buffer and sample channels. The objective lens was then used to focus a laser spot, measuring $\sim 8 \mu \mathrm{m}$ in diameter and using the $488 \mathrm{~nm}$ Ar laser line from a multi-line, $\mathrm{Ar} / \mathrm{Kr}$

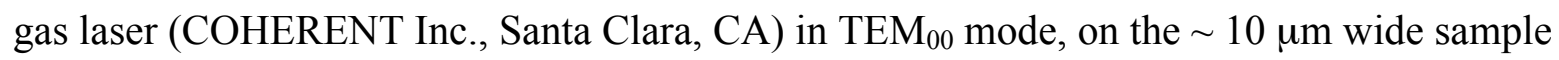
stream. The laser power was set at a constant $25 \mathrm{~mW}$. A dichroic mirror (U-N41001, Olympus) and $500 \mathrm{~nm}$ longpass emission filter (7512, Chroma Technology Corp., Rockingham, VT) were used to separate the excitation light from the collected fluorescence. Fluorescence emission was separated into spectral bands using dirchroic mirrors (q555lp and 610dlp, Chroma Technology Corp.) and bandpass filters before being focused on the active areas of solid-state photo detectors (see Figure 1A). For green wavelengths, a 500-540 nm bandpass filter (HQ520/40, Chroma Technology Corp.) was used with a bi-convex lens (LB1761-A, Thorlabs Inc., Newton, NJ) to focus these emissions on a PIN photodiode detector (818 Series, Newport Corp., Irvine, CA). This detector was connected to an optical power meter (1830-C, Newport Corp.). Similarly, bandpass filters (HQ575/30 and HQ630/60, Chroma Technology Corp.) and lenses (LB1811-A and LA1027-A, respectively, Thorlabs Inc.) were used for two avalanche photodiodes (C4777-01 
and C5460 for yellow and red channels, respectively, Hamamatsu Corp., Bridgewater, NJ). Voltage outputs for all three detectors were connected to ports of a data acquisition card (NI USB-6251, National Instruments, Austin, TX) that was relayed to a computer and operated using Labview software (National Instruments). Sampling at $1 \mathrm{kHz}$ was used, though the capabilities of the data acquisition card are on the order of $1 \mathrm{MS} / \mathrm{s}$. During an experiment, voltages from the three detectors were output into .txt data files with four columns of data, the first column listing the time, while the three adjacent columns listed the green, yellow and red bandpass detector voltages, respectively.

A typical experiment ran for 15 minutes, allowing the collection of $\sim 30 \mathrm{MB}$ of data and $\sim 1000$ detection events. After an experiment, the QdotB complexes remaining in the sample channel were collected and counted using a cell counter (Vi-Cell XR, Beckman-Coulter, Fullerton, CA) while the microchannel was disposed of. QdotB complex concentrations in the sample well were $1.5 \times 10^{7} / \mathrm{mL}\left(9 \times 10^{6}\right.$ standard deviation $)$ during a typical experiment.

\section{Collection of Spectra Using a CCD Array Camera}

The collection of spectra such as those shown in Figure 2C used similar steps to those given in section 6. Fluorescence emission, however, was directed towards and focused on the entrance slit of a spectrograph (Acton Research Inc., Acton, MA) using a $50.0 \mathrm{~mm}$ focal length lens (LA1131-A, Thorlabs Inc.). Inside the spectrograph a grating with 150 grooves per mm was used to disperse the emission light by wavelength before illuminating the pixels of a thermoelectrically cooled CCD camera (7481-0002, Princeton Instruments, Trenton, NJ). The selection of this grating allowed inspection of the spectrum from $\sim 450-700 \mathrm{~nm}$ when aligned for a central wavelength of $570 \mathrm{~nm}$. 
The integration time of the CCD array was $50 \mathrm{msec}$, set by a mechanical shutter, while typical readout time was $\sim 250$ msec. Data taken by the camera was collected and analyzed using WinSpec software (Princeton Instruments). By collecting multiple, successive spectra, it was possible to discriminate background and detection signals from each other.

\section{Images of QdotB Microbeads}

The images shown in Figures 1B and 2A were taken using an inverted epifluorescent microscope (IX71, Olympus) with a Hg lamp excitation source (BH2-RFL-T3, Olympus). A UV excitation filter (D350/50, Chroma Technology Corp.) and $430 \mathrm{~nm}$ longpass emission filter (HQ430LP, Chroma Technology Corp.) were used in combination with a dichroic mirror (UN31000, Olympus) and 40X microscope objective (0.75 NA, Olympus). Samples were imaged in solution using a cell chamber (A-7816, Invitrogen) and $25 \mathrm{~mm}$ diameter, $170 \mu \mathrm{m}$ thick coverslips (VWR).

\section{Quantum Dot Barcode Detection Algorithm}

\subsection{Dilution Sensitivity Experiments}

As mentioned in section 6, many data files from a detection experiment were collected in .txt format that contained 4 columns of data: time, and the three temporal detector output voltages. These data files were loaded by analysis programs developed in house using MATLAB $^{\mathrm{TM}}$ software (MathWorks, Natick, MA). First, the detector voltages were converted to absolute values (voltage values were either all positive or all negative, with detection peaks oriented in more positive or negative directions, respectively) and then smoothed using a moving average filter twice (of length 9). Baseline offset values were determined for each set of detector 
voltages; these values were calculated by taking the mean of all sample points smaller than the overall mean of all the sample points.

Thresholds were evaluated for each relevant set of detector voltages depending on the experiment (e.g., for HBV experiments, the green and yellow channel values were only of concern) by calculating the mean of sample points greater than the overall mean of the baseline offset. Location, full width at half maximum (FWHM) and duration of each peak exceeding the threshold criteria were evaluated. Peaks which fulfilled the previous requirements (such as simultaneous peak detection in the green and yellow channels for HBV experiments) were then converted to normalized quantities $\langle G\rangle,\langle Y\rangle$ and $\langle R\rangle$ for the green, yellow and red channels, respectively.

Peak data which passed previous scrutiny was subjected to two further screening procedures: a) The average and standard deviation of peak durations were calculated, and only those peaks falling within \pm one standard deviation of this mean were selected as valid peaks. b) Outlier analysis of normalized peak values was completed to remove any abnormally high or low peak values.

The remaining normalized green channel values were then averaged (for the results from three separate experiments using three different microfluidic chips and three samples of QdotB complexes) yielding the values given in Figures 3, $S 2$ and Tables $S 1$ and $S 2$. Standard deviation and count numbers for the experiments are also given in Tables $S 1$ and $S 2$.

\subsection{Multiplexed Detection Experiments}

For the multiplexed data analysis, preliminary normalized peak values for the green, yellow and red channels were screened as described in section 8.1. However, as a means of classifying the detection events for specific pathogens, the ratio of $\langle R\rangle /\langle Y\rangle$ had to be calculated 
for each peak. The distribution of $\langle R\rangle /\langle Y\rangle$ values was then plotted in a histogram and appropriate thresholds set for HBV, HIV and HCV populations, listed in order of increasing $\langle R\rangle /\langle Y\rangle$ value (see Figure 4A). $\langle G\rangle$ values were classified and grouped into HBV, HIV and HCV regions, yielding $\langle\bar{G}\rangle$ values for each group. The results are seen in Figures 4C and 4D. These values are also listed in Tables $S 3$ and $S 4$. 


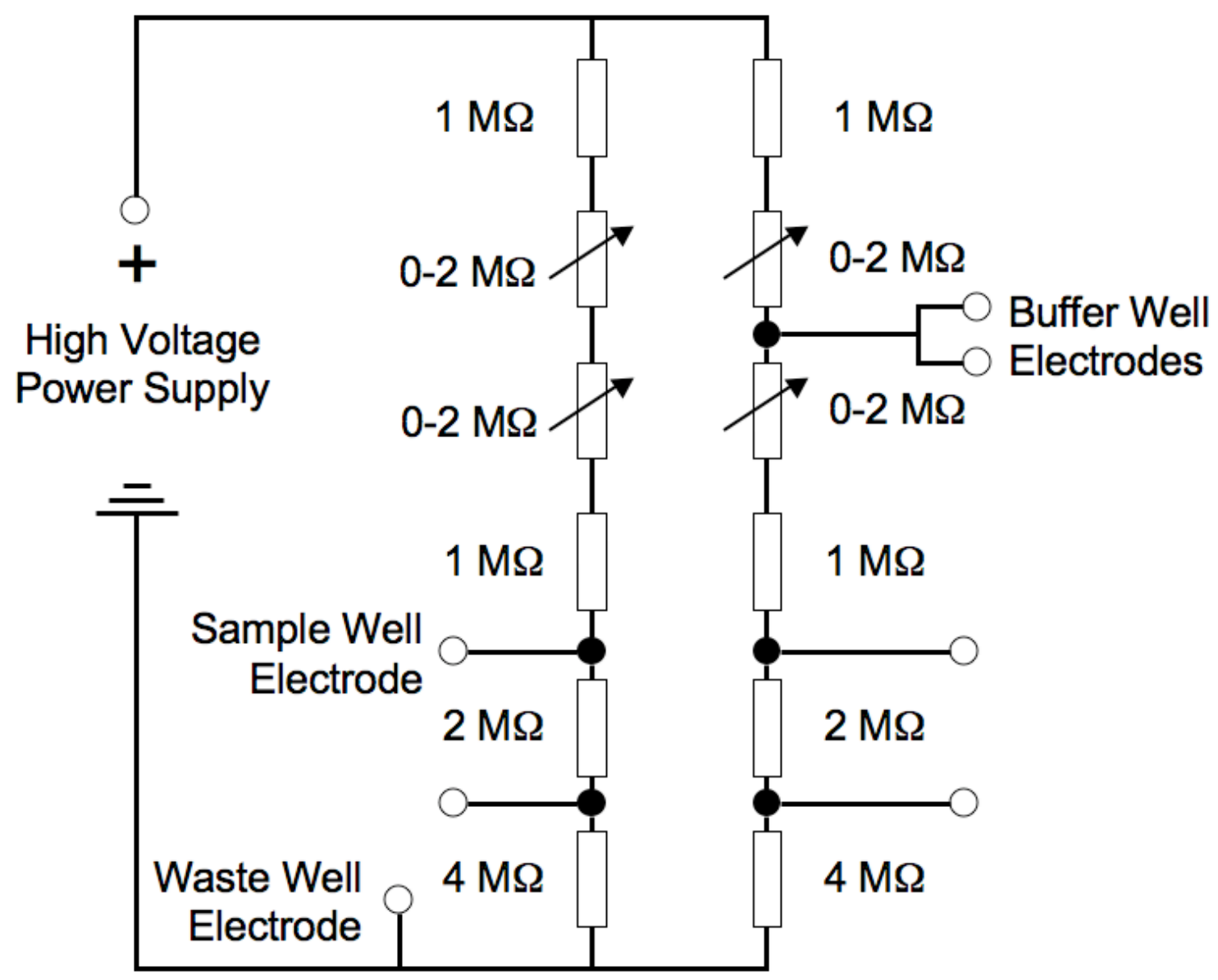

Figure S1: Voltage regulation circuit for electrokinetic flow focusing. Arrows indicate variable resistances that could be modulated to control sample flow stream width. 


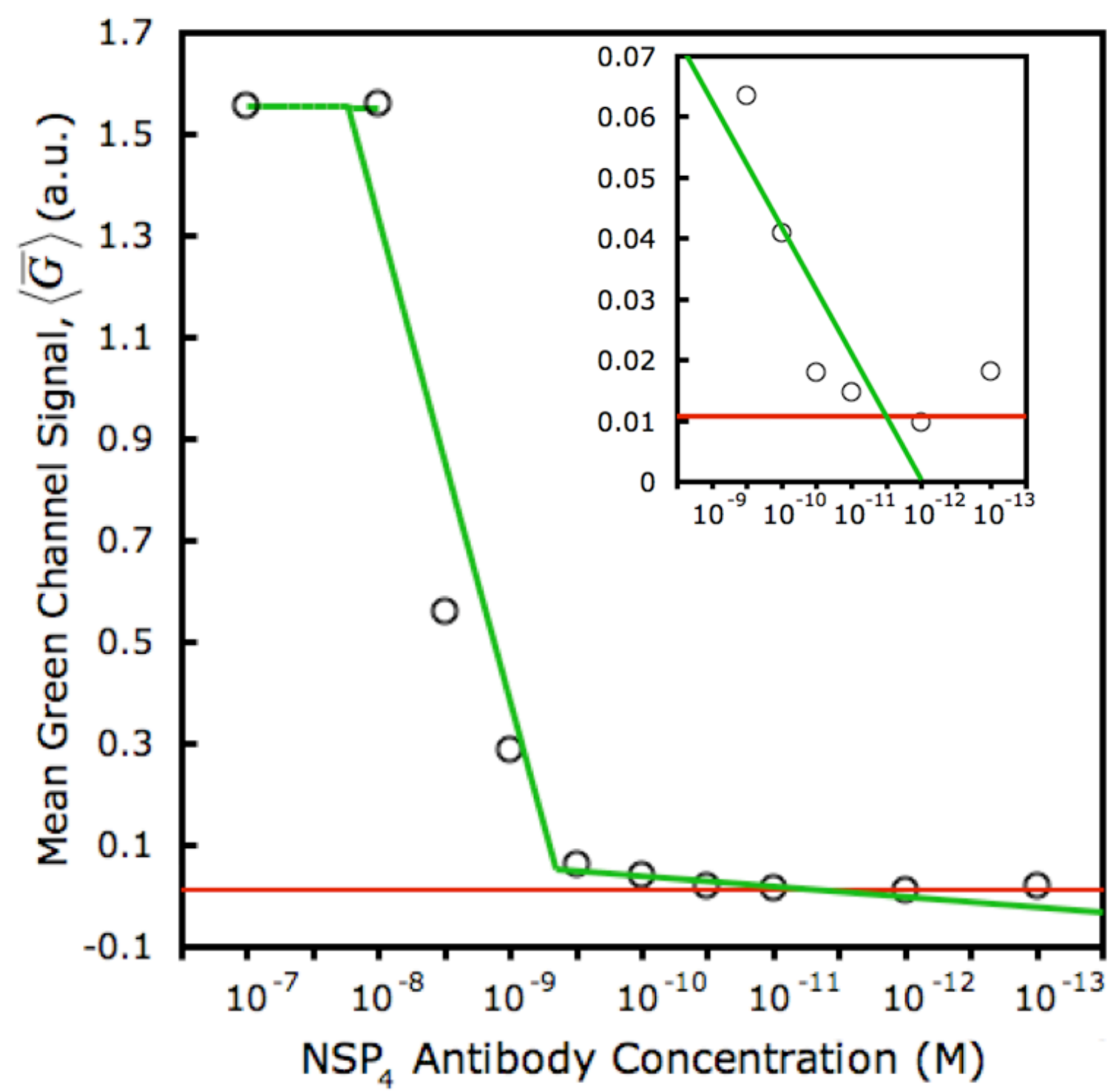

Figure $S 2$ : Dilution sensitivity curve for non-structural protein $4\left(\mathrm{NSP}_{4}\right)$ antibodies and hepatitis $\mathrm{C}$ virus. Mean green channel values, $\langle\bar{G}\rangle$, are given for a range of dilution levels of anit-NSP 4 in human serum and are fit with solid lines corresponding to the three different linear ranges present. The red horizontal line corresponds to the mean plus three times the $95 \%$ confidence interval of $\langle\bar{G}\rangle$ values for control (no anti-NSP 4 antibodies) experiments. The inset shows a detection sensitivity of $10^{-11.5} \mathrm{M}$ for anti-NSP${ }_{4}$. 


\section{SECTION S3 (MOVIE)}

A video of showing the flow profile in the flow focusing region using rhodamine $6 \mathrm{G}$ dye in double distilled water (part 1), the flow of quantum dot barcoded (QdotB) beads before entering (part 2) and after entering (part 3) the flow focusing region, and at the detection site (part 4). The QdotBs were excited by a $\mathrm{Hg}$ lamp (340 to $360 \mathrm{~nm}$ ) and the signal collected using a $40 \mathrm{x}$ objective (0.8 N.A.). The signal passes through a long pass filter (450 nm) and the video collected using an Olympus C-5050 camera. 


\section{SECTION S4 (TABLES)}

Table S1: Results of dilution detection experiments. Values are culminated for three separate experiments using three different microfluidic chips and three separately prepared samples. $\langle\bar{G}\rangle$

- mean normalized green channel signal, $s_{\langle\bar{G}\rangle}$ - standard deviation of mean normalized green channel signal, $N$ - number of detection events

\begin{tabular}{|c|c|c|c|c|c|}
\hline & $\begin{array}{l}\text { Sample } \\
\quad \text { Antibody } \\
\text { Concentration (M) }\end{array}$ & $\langle\bar{G}\rangle$ (a.u.) & $s_{\langle\bar{G}\rangle}$ (a.u.) & $N$ & $\begin{array}{c}95 \% \\
\text { Confidence } \\
\text { Interval }\end{array}$ \\
\hline 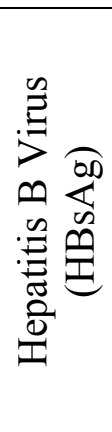 & $\begin{array}{c}9.47 \times 10^{-8} \\
9.47 \times 10^{-9} \\
4.74 \times 10^{-9} \\
9.47 \times 10^{-10} \\
4.74 \times 10^{-10} \\
9.47 \times 10^{-11} \\
4.74 \times 10^{-11} \\
9.47 \times 10^{-12} \\
9.47 \times 10^{-13} \\
\text { Control (No Ab) }\end{array}$ & $\begin{array}{l}1.6613 \\
1.6643 \\
1.0296 \\
0.2884 \\
0.2241 \\
0.1413 \\
0.1055 \\
0.0976 \\
0.0987 \\
0.0711\end{array}$ & $\begin{array}{l}0.1319 \\
0.1095 \\
0.1238 \\
0.0609 \\
0.0325 \\
0.0207 \\
0.0147 \\
0.0141 \\
0.0140 \\
0.0112\end{array}$ & $\begin{array}{l}2532 \\
1675 \\
2938 \\
2758 \\
2122 \\
1762 \\
2677 \\
1751 \\
1896 \\
2236\end{array}$ & $\begin{array}{l}0.0051 \\
0.0052 \\
0.0045 \\
0.0023 \\
0.0014 \\
0.0010 \\
0.0006 \\
0.0007 \\
0.0006 \\
0.0005\end{array}$ \\
\hline 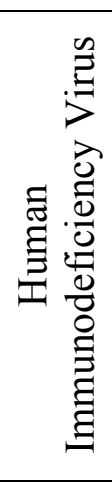 & $\begin{array}{c}9.47 \times 10^{-8} \\
9.47 \times 10^{-9} \\
4.74 \times 10^{-9} \\
9.47 \times 10^{-10} \\
4.74 \times 10^{-10} \\
9.47 \times 10^{-11} \\
4.74 \times 10^{-11} \\
9.47 \times 10^{-12} \\
9.47 \times 10^{-13} \\
9.47 \times 10^{-14} \\
\text { Control (No } \mathrm{Ab})\end{array}$ & $\begin{array}{l}1.1898 \\
0.7790 \\
0.5283 \\
0.2399 \\
0.1206 \\
0.0592 \\
0.0420 \\
0.0313 \\
0.0177 \\
0.0300 \\
0.0228 \\
\end{array}$ & $\begin{array}{l}0.1630 \\
0.2060 \\
0.0802 \\
0.0375 \\
0.0164 \\
0.0101 \\
0.0094 \\
0.0069 \\
0.0072 \\
0.0097 \\
0.0080 \\
\end{array}$ & $\begin{array}{l}1974 \\
1388 \\
1924 \\
1695 \\
2119 \\
1905 \\
2212 \\
2109 \\
2163 \\
1964 \\
2048 \\
\end{array}$ & $\begin{array}{l}0.0072 \\
0.0108 \\
0.0036 \\
0.0018 \\
0.0007 \\
0.0005 \\
0.0004 \\
0.0003 \\
0.0003 \\
0.0004 \\
0.0003 \\
\end{array}$ \\
\hline 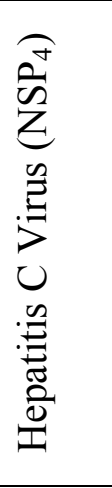 & $\begin{array}{c}9.47 \times 10^{-8} \\
9.47 \times 10^{-9} \\
4.74 \times 10^{-9} \\
9.47 \times 10^{-10} \\
4.74 \times 10^{-10} \\
9.47 \times 10^{-11} \\
4.74 \times 10^{-11} \\
9.47 \times 10^{-12} \\
9.47 \times 10^{-13} \\
9.47 \times 10^{-14} \\
\text { Control }(\mathrm{No} \mathrm{Ab})\end{array}$ & \begin{tabular}{l|}
.5562 \\
1.5600 \\
0.5599 \\
0.2891 \\
0.0635 \\
0.0409 \\
0.0180 \\
0.0147 \\
0.0097 \\
0.0181 \\
0.0104 \\
\end{tabular} & $\begin{array}{c}0.1009 \\
0.1013 \\
0.0621 \\
0.034 \\
0.0074 \\
0.0126 \\
0.0050 \\
0.0028 \\
0.0028 \\
0.0223 \\
0.0040 \\
\end{array}$ & $\begin{array}{l}467 \\
2381 \\
2987 \\
3583 \\
2421 \\
1552 \\
3199 \\
2251 \\
3427 \\
3813 \\
3019 \\
\end{array}$ & $\begin{array}{l}0.0030 \\
0.0041 \\
0.0022 \\
0.0011 \\
0.0003 \\
0.0006 \\
0.0002 \\
0.0001 \\
0.0001 \\
0.0007 \\
0.0001 \\
\end{array}$ \\
\hline
\end{tabular}


Table S2: Results from FDA-approved HBsAg ELISA kit (Biorad). Kit protocols required three negative controls, two positive controls and three samples be measured for optical density after the assay. The control sample optical densities determine the detection cutoff value. Listed are the target antibody concentrations in human serum, the average positive control optical density, $\bar{x}_{\text {positive }}$, the average negative control optical density, $\bar{x}_{\text {negative }}$, the corresponding detection cutoff value, the average sample optical density, $\bar{x}_{\text {sample }}$, and its standard deviation, $s_{\text {sample }}$. The last column lists whether the detection was positive or negative according to kit protocols.

\begin{tabular}{ccccccc}
\hline $\begin{array}{c}\text { Sample } \\
\begin{array}{c}\text { Antibody } \\
\text { Concentration } \\
(\mathrm{M})\end{array}\end{array}$ & $\begin{array}{c}\bar{x}_{\text {positive }} \\
(\text { a.u. })\end{array}$ & $\begin{array}{c}\bar{x}_{\text {negative }} \\
\text { (a.u.) }\end{array}$ & $\begin{array}{c}\text { Cutoff } \\
\text { Value } \\
\text { (a.u.) }\end{array}$ & $\begin{array}{c}\bar{x}_{\text {sample }} \\
(\text { a.u. })\end{array}$ & $\begin{array}{c}S_{\text {sample }} \\
(\text { a.u. })\end{array}$ & Detection \\
\hline $9.47 \times 10^{-8}$ & 0.9725 & 0.0588 & 0.1338 & 2.9572 & 0.3153 & Positive \\
$9.47 \times 10^{-9}$ & 0.9683 & 0.0450 & 0.1200 & 2.2164 & 0.1454 & Positive \\
$4.74 \times 10^{-9}$ & 0.9090 & 0.0484 & 0.1234 & 1.7084 & 0.1845 & Positive \\
$9.47 \times 10^{-10}$ & 0.9385 & 0.0472 & 0.1222 & 0.8496 & 0.1131 & Positive \\
$4.74 \times 10^{-10}$ & 0.9103 & 0.0472 & 0.1222 & 0.2000 & 0.0214 & Positive \\
$9.47 \times 10^{-11}$ & 0.9198 & 0.0491 & 0.1241 & 0.0877 & 0.0084 & Negative \\
$4.74 \times 10^{-11}$ & 0.8818 & 0.0500 & 0.1250 & 0.0628 & 0.0086 & Negative \\
$9.47 \times 10^{-12}$ & 0.8547 & 0.0496 & 0.1246 & 0.0582 & 0.0117 & Negative \\
$9.47 \times 10^{-13}$ & 0.8002 & 0.0529 & 0.1279 & 0.0547 & 0.0040 & Negative \\
\hline
\end{tabular}


Table S3: Results from FDA-approved gp41 ELISA kit (Biorad). Kit protocols required three negative controls and three positive controls be measured for optical density after the assay to determine the detection cutoff value. Three samples were also to be tested for each target antibody concentration. Listed are the target antibody concentrations in human serum, the average positive control optical density, $\bar{x}_{\text {positive }}$, the average negative control optical density, $\bar{x}_{\text {negative }}$, the corresponding detection cutoff value, the average sample optical density, $\bar{x}_{\text {sample }}$, and its standard deviation, $s_{\text {sample }}$. The last column lists whether the detection was positive or negative according to kit protocols.

\begin{tabular}{ccccccc}
\hline $\begin{array}{c}\text { Sample } \\
\begin{array}{c}\text { Antibody } \\
\text { Concentration } \\
(\mathrm{M})\end{array}\end{array}$ & $\begin{array}{c}\bar{x}_{\text {positive }} \\
\text { (a.u.) }\end{array}$ & $\begin{array}{c}\bar{x}_{\text {negative }} \\
\text { (a.u.) }\end{array}$ & $\begin{array}{c}\text { Cutoff } \\
\text { Value } \\
\text { (a.u.) }\end{array}$ & $\begin{array}{c}\bar{x}_{\text {sample }} \\
\text { (a.u.) }\end{array}$ & $\begin{array}{c}s_{\text {sample }} \\
\text { (a.u.) }\end{array}$ & Detection \\
\hline $9.47 \times 10^{-8}$ & 1.697 & 0.189 & 0.439 & 3.924 & 0.021 & Positive \\
$9.47 \times 10^{-9}$ & 1.697 & 0.189 & 0.439 & 3.932 & 0.079 & Positive \\
$4.74 \times 10^{-9}$ & 1.697 & 0.189 & 0.439 & 3.976 & 0.014 & Positive \\
$9.47 \times 10^{-10}$ & 1.697 & 0.189 & 0.439 & 3.706 & 0.050 & Positive \\
$4.74 \times 10^{-10}$ & 1.697 & 0.189 & 0.439 & 2.249 & 0.097 & Positive \\
$9.47 \times 10^{-11}$ & 1.697 & 0.189 & 0.439 & 0.992 & 0.046 & Positive \\
$4.74 \times 10^{-11}$ & 1.697 & 0.189 & 0.439 & 0.403 & 0.028 & Negative \\
$9.47 \times 10^{-12}$ & 1.697 & 0.189 & 0.439 & 0.286 & 0.077 & Negative \\
$9.47 \times 10^{-13}$ & 1.697 & 0.189 & 0.439 & 0.155 & 0.028 & Negative \\
$9.47 \times 10^{-14}$ & 1.697 & 0.189 & 0.439 & 0.162 & 0.020 & Negative \\
\hline
\end{tabular}


Table S4: Results of multiplexed detection experiments. Values are culminated for three separate experiments using three different microfluidic chips and three separately prepared samples. $\langle R\rangle /\langle Y\rangle$ signal ratios were used to classify detection events as HBV, HIV or HCV (see Figure 4). Listed below are: $\langle\bar{G}\rangle_{\mathrm{HBV}},\langle\bar{G}\rangle_{\mathrm{HIV}},\langle\bar{G}\rangle_{\mathrm{HCV}}$ - the mean normalized green channel signals for HBV, HIV and HCV detection events, respectively; $s_{\langle\bar{G}\rangle_{\mathrm{HBV}}}, s_{\langle\bar{G}\rangle_{\mathrm{HIV}}}, s_{\langle\bar{G}\rangle_{\mathrm{HCV}}}$ - the standard deviations of the mean normalized green channel signals for HBV, HIV and HCV, respectively; $N_{\mathrm{HBV}}, N_{\mathrm{HIV}}, N_{\mathrm{HCV}}$ - the number of counts for $\mathrm{HBV}$, HIV and HCV, respectively. For Figures 4C and 4D, the values listed in the table were normalized with respect to the largest value in each column to help visualize the differences in detection signal intensity.

\begin{tabular}{|c|c|c|c|c|c|c|c|c|c|c|}
\hline & Sample & $\langle\bar{G}\rangle_{\mathrm{HBV}}$ & $\langle\bar{G}\rangle_{\mathrm{HIV}}$ & $\langle\bar{G}\rangle_{\mathrm{HCV}}$ & $s_{\langle\bar{G}\rangle_{\mathrm{HBV}}}$ & $s_{\langle\bar{G}\rangle_{\mathrm{HIV}}}$ & $s_{\langle\bar{G}\rangle_{\mathrm{HCV}}}$ & $N_{\mathrm{HBV}}$ & $N_{\text {HIV }}$ & $N_{\mathrm{HCV}}$ \\
\hline \multirow{8}{*}{ 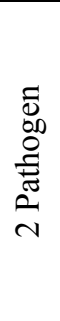 } & $\mathrm{HBV}+, \mathrm{HIV}+$ & 1.204 & 0.251 & - & 0.051 & 0.019 & - & 3427 & 1368 & - \\
\hline & $\mathrm{HBV}+, \mathrm{HIV}-$ & 1.326 & 0.019 & 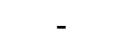 & 0.075 & 0.009 & - & 3959 & 1981 & 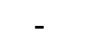 \\
\hline & HBV-,HIV+ & 0.019 & 0.436 & - & 0.011 & 0.037 & - & 2180 & 1863 & - \\
\hline & HBV-,HIV- & 0.020 & 0.015 & - & 0.004 & 0.003 & - & 1706 & 1989 & - \\
\hline & Control 1 & 0.016 & 0.020 & - & 0.002 & 0.002 & - & 675 & 1986 & - \\
\hline & Control 2 & 0.027 & 0.019 & - & 0.005 & 0.004 & - & 3200 & 3084 & - \\
\hline & Control 3 & 0.018 & 0.019 & - & 0.004 & 0.004 & - & 1922 & 3179 & - \\
\hline & Control 4 & 0.017 & 0.011 & - & 0.002 & 0.001 & - & 2756 & 3026 & - \\
\hline \multirow{10}{*}{$\begin{array}{l}\bar{D} \\
\text { on } \\
0 \\
\text { है } \\
2 \\
m\end{array}$} & $\mathrm{HBV}+, \mathrm{HIV}+, \mathrm{HCV}+^{+}$ & 1.350 & 0.384 & 0.227 & 0.129 & 0.061 & 0.021 & 1090 & 1088 & 774 \\
\hline & HBV-,HIV+,HCV+ & 0.030 & 0.338 & 0.198 & 0.009 & 0.057 & 0.027 & 211 & 980 & 861 \\
\hline & $\mathrm{HBV}+, \mathrm{HIV}+, \mathrm{HCV}-$ & 1.467 & 0.438 & 0.031 & 0.125 & 0.102 & 0.008 & 1458 & 394 & 689 \\
\hline & $\mathrm{HBV}+, \mathrm{HIV}-, \mathrm{HCV}+$ & 1.477 & 0.065 & 0.153 & 0.108 & 0.022 & 0.023 & 1631 & 450 & 460 \\
\hline & HBV-,HIV-,HCV- & 0.019 & 0.017 & 0.014 & 0.008 & 0.005 & 0.003 & 1092 & 1933 & 920 \\
\hline & Control 1 & 0.034 & 0.019 & 0.024 & 0.014 & 0.005 & 0.003 & 115 & 1300 & 527 \\
\hline & Control 2 & 0.042 & 0.035 & 0.014 & 0.017 & 0.006 & 0.004 & 167 & 1288 & 835 \\
\hline & Control 3 & 0.025 & 0.029 & 0.024 & 0.008 & 0.004 & 0.003 & 549 & 1481 & 824 \\
\hline & Control 4 & 0.037 & 0.021 & 0.019 & 0.007 & 0.005 & 0.003 & 139 & 438 & 312 \\
\hline & Control 5 & 0.014 & 0.010 & 0.008 & 0.003 & 0.002 & 0.001 & 492 & 1862 & 821 \\
\hline
\end{tabular}




\section{SECTION S5 (COST-ANALYSIS)}

\section{Approximating the cost analysis for a single assay (assuming the instrument is already}

purchased). The following approximates the operational costs of the platform per sample and compares it to the cost for conventional ELISA kits. We compare our costs to test for HBsAg with the cost to do the same with the commercial kit used. The operational costs are assumed to be those for the consumables needed for a detection experiment. The consumables for a typical experiment considered are PDMS microfluidic chips, polystyrene microbeads, quantum dots, pathogen antigens and detection antibodies conjugated to fluorescent dye. Canadian currency is assumed (1.20 Canadian Dollars $=1.00$ United States Dollar).

The cost of a typical PDMS microfluidic chip was rationalized to be about $\$ 0.50$. If we assume the cost of 1 gallon of PDMS prepolymer to be about $\$ 350.00$, and we use $20 \mathrm{~g}$ to make $\sim 4$ microfluidic chips, then the cost of PDMS is $\$ 0.48$ (assuming a PDMS density of 965 $\mathrm{kg} / \mathrm{m}^{3}$ ). If coverslips are assumed at a cost of several cents, then the assumption of $\$ 0.50$ per PDMS microfluidic chip is reasonable.

In this section, we describe the cost of preparing a large batch of targeted QdotBs (which corresponds to $~ 3.7$ million QdotBs; for each assay, only 2,000 QdotBs are used). $\quad 5 \mu \mathrm{m}$ diameter polystyrene microbeads cost $\sim \$ 500$ for $1.0 \mathrm{~g}$, assuming $10 \%$ solids. At a bead concentration of $1.5 \times 10^{9}$ microbeads $/ \mathrm{mL}$, our protocol uses $2.5 \mu \mathrm{L}$ per sample. Since $1.0 \mathrm{~g}$ of microbeads ( $10 \%$ solids) is nearly $1 \mathrm{~mL}$ in volume, we can assume the microbead cost to be $\$ 1.25$ per preparation.

Quantum dots can be purchased for a cost of roughly $\$ 750$ per $200 \mathrm{mg}$ (Evident Technologies Inc., Troy, NY). A typical synthesis produces $500 \mathrm{mg}$, and in our case, $\sim 40 \mathrm{~mL}$ of Qdot in chloroform. We used $<100 \mu \mathrm{L}$ to dope our microbeads, which means for a $200 \mathrm{mg}$ 
sample, it should be possible to dope $\sim 160$ samples. This make the Qdot cost per preparation $\sim$ $\$ 4.50$.

Antigen solution for HBsAg costs $\sim \$ 300$ for a $0.5 \mathrm{~mL}$ sample. This solution was diluted 1:25 times and since a single sample requires $100 \mu \mathrm{L}, 4 \mu \mathrm{L}$ of stock solution is used per sample. With a single bottle of antigen solution being sufficient for 125 samples, its cost per preparation is $\$ 2.40$.

Finally, the detection antibody solution (goat anti-mouse IgG conjugated to AlexaFluor488 dye) was purchased at a cost of $\sim \$ 220$ for $0.5 \mathrm{~mL}$. Since this solution is diluted 300 times, $0.3 \mu \mathrm{L}$ of stock solution was used per preparation at a cost of $\sim \$ 0.15$.

Adding all the consumable costs together yields a cost of $\$ 8.80$ per preparation of 3.7 million QdotBs. For each assay, only $\sim 2,000$ QdotBs are used. Therefore, the actual cost for the QdotBs is $<\$ 0.01$. The real cost for the assay stems from only the microfluidic chip, which is $\$ 0.50$.

The ELISA kit we used cost $\sim \$ 350$ for a 96 well plate and reagents. For a single diagnosis eight of these wells are required (three for negative controls, two for positive controls and three for sample). Therefore, the cost of a single diagnosis is $\$ 29.17$.

Although we have shown the cost to be less for consumables using the platform over ELISA, we have also assumed to be using commercial microbeads and Qdots. However, if we supply our microbeads, we can drop those costs to less than $\$ 0.10$ per preparation. Furthermore, we synthesize our own Qdots at a cost of $\sim \$ 30.00$ per batch $(500 \mathrm{mg})$. This means our Qdot cost per preparation is also $<\$ 0.10$. This would make this microbead-based assay system even more cost-effective. 


\section{References}

S1. Murray, C. B., Norris, D. J., Bawendi, M. G., J. Am. Chem. Soc., 1993, 115, 8706.

S2. Hines, M. A., Guyot-Sionnest, P., J. Phys. Chem., 1996, 100, 468.

S3. Dabbousi, B. O., Rodriguez-Viejo, J., Mikulec, F. V., Heine, J. R., Mattoussi, H., Ober, R., Jensen, K. F., Bawendi, M. G., J. Phys. Chem. B, 1997, 101, 9463.

S4. Peng, X., Schlamp, M. C., Kadavanich, A. V., Alivisatos, A. P., J. Am. Chem. Soc., 1997, 119, 7019.

S5. Han, M., Gao, X., Su, J. Z., Nie, S., Nat. Biotech., 2001, 19, 631.

S6. Gao, X., Nie, S., Anal. Chem., 2004, 76, 2406.

S7. Xia, Y., Whitesides, G. M., Annu. Rev. Mater. Sci., 1998, 28, 153.

S8. Duffy, D. C., McDonald, J. C., Schueller, O. J. A., Whitesides, G. M., Anal. Chem., 1998, 70, 4974.

S9. Unger, M. A., Chou, H., Thorsen, T., Scherer, A., Quake, S. R., Science, 2000, 288, 113.

S10. Mekler, V. M., Bystryak, S. M., Anal. Chim. Acta, 1992, 264, 359.

S11. Tarcha, P. J., Chu, V. P., Whittern, D., Anal. Biochem., 1987, 165, 230. 